\title{
On the Lack of Type I X-Ray Bursts in Black Hole X- Ray Binaries: Evidence for the Event Horizon?
}

\section{Citation}

Narayan, Ramesh, and Jeremy S. Heyl. 2002. "On the Lack of Type I X-Ray Bursts in Black Hole X-Ray Binaries: Evidence for the Event Horizon?" The Astrophysical Journal 574 (2): L139-42. https://doi.org/10.1086/342502.

\section{Permanent link}

http://nrs.harvard.edu/urn-3:HUL.InstRepos:41384943

\section{Terms of Use}

This article was downloaded from Harvard University's DASH repository, and is made available under the terms and conditions applicable to Other Posted Material, as set forth at http:// nrs.harvard.edu/urn-3:HUL.InstRepos:dash.current.terms-of-use\#LAA

\section{Share Your Story}

The Harvard community has made this article openly available.

Please share how this access benefits you. Submit a story.

Accessibility 


\title{
On the Lack of Type I X-ray Bursts in Black Hole X-ray Binaries: Evidence for the Event Horizon?
}

\author{
Ramesh Narayan ${ }^{1,2}$ \& Jeremy S. Heyl ${ }^{2,3}$
}

\begin{abstract}
Type I X-ray bursts are very common in neutron star X-ray binaries, but no Type I burst has been seen in the dozen or so binaries in which the accreting compact star is too massive to be a neutron star and therefore is identified as a black hole candidate. We have carried out a global linear stability analysis of the accumulating fuel on the surface of a compact star to identify the conditions under which thermonuclear bursts are triggered. Our analysis, which improves on previous calculations, reproduces the gross observational trends of bursts in neutron star systems. It further shows that, if black hole candidates have surfaces, they would very likely exhibit instabilities similar to those that lead to Type I bursts on neutron stars. The lack of bursts in black hole candidates is thus significant, and indicates that these objects have event horizons. We discuss possible caveats to this conclusion.
\end{abstract}

Subject headings: accretion — black hole physics — X-rays: binaries, bursts

\section{Introduction}

When gas accretes onto a neutron star (NS) in a low-mass X-ray binary (LMXB), it is compressed and heated as it accumulates on the surface, leading to thermonuclear reactions. In many NS LMXBs, the reactions occur unsteadily and cause Type I X-ray bursts (Grindlay et al. 1976). Type I bursts have been observed in a large number of NS LMXBs (see Lewin, van Paradijs \& Taam 1993; Strohmayer, Swank \& Zhang 1998 for reviews), and the theory of these bursts is relatively well understood (Hansen \& van Horn 1975; Woosley \& Taam

\footnotetext{
${ }^{1}$ Institute for Advanced Study, Princeton, NJ 08540

${ }^{2}$ Harvard-Smithsonian Center for Astrophysics, Cambridge, MA 02138; rnarayan@cfa.harvard.edu; jheyl@cfa.harvard.edu

${ }^{3}$ Chandra Fellow
} 
1976; Joss 1977; Taam \& Picklum 1978; Paczyński 1982, hereafter P82; Fujimoto et al. 1984, 1987; Fushiki \& Lamb 1987, hereafter FL87; Taam, Woosley \& Lamb 1996; Bildsten 1998).

Among the over 100 LMXBs known in the Galaxy, roughly a dozen systems, most of them transient sources (Tanaka \& Shibazaki 1996), have been identified as black hole (BH) candidates. In these BH LMXBs, dynamical measurements give mass estimates for the accreting stars greater than the likely maximum mass $\sim 3 M_{\odot}$ of a NS (Shapiro \& Teukolsky 1983, hereafter ST83; Narayan, Garcia \& McClintock 2001; and references therein).

No Type I burst has been seen in a BH LMXB, even though, as we show in this Letter, BH LMXBs ought to produce bursts as efficiently as NS LMXBs if the accreting $\mathrm{BH}$ candidates possess surfaces. The lack of bursts thus represents possible evidence for the presence of event horizons. In $\S 2$ of this paper, we examine the stability of nuclear burning on the surface of a compact star. In $\S 3$, we discuss the predictions of the model for accretion onto a $1.4 M_{\odot} \mathrm{NS}$ and a $10 M_{\odot} \mathrm{BH}$ candidate with a hypothetical surface. We conclude in $\S 4$ with a discussion.

\section{The Model}

We consider a compact spherical star of mass $M$ and radius $R$, accreting gas steadily at a rate $\dot{\Sigma}\left(\mathrm{g} \mathrm{cm}^{-2} \mathrm{~s}^{-1}\right)$. In the local frame, the gravitational acceleration is $g=G M(1+$ $z) / R^{2}$, where the redshift $z$ is given by $1+z=\left(1-R_{S} / R\right)^{-1 / 2}$, and $R_{S}=2 G M / c^{2}$ is the Schwarzschild radius. We assume that the accreting material has mass fractions $X_{0}, Y_{0}$ and $Z_{0}=1-X_{0}-Y_{0}$, of hydrogen, helium and heavier elements (mostly CNO).

We consider a layer of accreted material of surface density $\Sigma_{\max }$ sitting on top of a substrate of fully burnt material $(X=Y=0, Z=1)$. (The particular composition of the substrate is unimportant since we apply the inner boundary condition at its top.) Since the physical thickness of the accreted layer is much less than the radius, we work in plane parallel geometry and take $g$ to be independent of depth. We solve for the density $\rho$, the temperature $T$, the outgoing flux $F$, and the hydrogen, helium and heavy element fractions, $X, Y, Z=1-X-Y$, as functions of the column density $\Sigma\left(0 \leq \Sigma \leq \Sigma_{\max }\right)$.

The evolution equations for the gas in the layer are the standard equations of stellar physics. H- and He-burning give

$$
\frac{d X}{d t}=-\frac{\epsilon_{H}}{E_{H}^{*}}, \quad \frac{d Y}{d t}=\frac{\epsilon_{H}}{E_{H}^{*}}-\frac{\epsilon_{H e}}{E_{H e}^{*}},
$$

where for our problem the total time derivative takes the form $d / d t \equiv \partial / \partial t+\dot{\Sigma} \partial / \partial \Sigma$. Here, $\epsilon_{H, H e}$ are the respective nuclear energy generation rates, and $E_{H, H e}^{*}$ are the corresponding 
energy release per unit mass of $\mathrm{H}$ and He burned (P82). For $\epsilon_{H}$, we include the pp chain and the CNO cycle, including fast-CNO burning, saturated CNO burning, and electron capture reactions, as described in Mathews \& Dietrich (1984) and Bildsten \& Cumming (1998). Since we are not concerned with modelling the bursts themselves, and since our stability criterion does not depend on the detailed treatment of the deep crust, we do not include proton captures onto heavier nuclei; Schatz et al. (1999) illustrate some of the consequences of the $r p$-process burning on accreting neutron stars. For He-burning, we include the triple- $\alpha$ reaction, but not pycnonuclear reactions (e.g., ST83). We do not correct the reaction rates to include screening (e.g. FL87) since we are concerned only with determining whether nuclear burning of $\mathrm{H}$ and He can proceed stably under given conditions; stable burning of $\mathrm{H}$ and $\mathrm{He}$ utilizes almost exclusively the reactions included.

Hydrostatic equilibrium gives $\partial P / \partial \Sigma=g$. For the pressure $P$ we use the expressions given in P82 for the gas, radiation and degeneracy pressure, along with a correction when the degenerate electrons become relativistic. Radiative transfer gives $\partial T / \partial \Sigma=(3 \kappa F) /\left(16 \sigma T^{3}\right)$, where $\sigma$ is the Stefan-Boltzmann constant and we write the opacity $\kappa$ in the form $1 / \kappa=$ $1 / \kappa_{\text {rad }}+1 / \kappa_{\text {cond }}$. We employ Iben's (1975) fitting functions for the radiative opacity $\kappa_{\text {rad }}$, and an analytical formula from Clayton (1968), suitably modified for relativistic electrons, for the conductive opacity $\kappa_{\text {cond }}$; the latter expression agrees well with more modern treatments (e.g. Heyl \& Hernquist 2001). Finally, the energy equation gives

$$
\rho T \frac{d s}{d t}=\rho\left(\epsilon_{H}+\epsilon_{H e}\right)+\rho \frac{\partial F}{\partial \Sigma}
$$

where $s$ is the entropy per unit mass. The above five equations form a closed set.

We have four outer boundary conditions at the surface of the star $(\Sigma=0)$. Two of these are $(X, Y)=\left(X_{0}, Y_{0}\right)$. We obtain the third boundary condition by equating the accretion luminosity of the infalling gas, $L_{a c c}=4 \pi R^{2} \dot{\Sigma} c^{2} z /(1+z)$, to blackbody emission from the surface: $4 \pi R^{2} \sigma T_{\text {out }}^{4}=L_{\text {acc }}$. This gives the surface temperature $T_{\text {out }}$. Then, using $T_{\text {out }}$ and an assumed value of $F_{\text {out }}$, we solve for the surface density profile $\rho(\Sigma)$ from the radiative transfer equation, thus obtaining the fourth boundary condition.

At the base of the accreted layer we have an inner boundary condition. We assume that the temperature at the top of the substrate, $T_{i n}=T\left(\Sigma_{\max }\right)$, is fixed. We examine several values of $T_{i n}$ for layers with $\Sigma_{\max }=10^{9}, 10^{10}$ and $10^{11} \mathrm{~g} \mathrm{~cm}^{-2}$. Applying the boundary condition at $\Sigma_{\max }$ rather than deeper down is an approximation, but the error due to this is not serious. For the high surface densities we consider, the heat transfer is dominated by conduction (Heyl \& Hernquist 2001), so the temperature gradient for $\Sigma>\Sigma_{\max }$ is small. Moreover, we examine models for several values of $T_{i n}$ which further mitigates any error.

The calculations proceed in two stages. First, we solve for the steady state profile of the 
accretion layer by making the replacement $d / d t \rightarrow \dot{\Sigma} d / d \Sigma, \partial / \partial \Sigma \rightarrow d / d \Sigma$ in the governing equations. This gives five ordinary differential equations with four outer boundary conditions and one inner boundary condition. The solution gives the profiles of the basic fluid quantities: $X(\Sigma), Y(\Sigma), P(\Sigma)=g \Sigma, \rho(\Sigma), T(\Sigma)$.

Having calculated the steady state structure of the accretion layer, we next check its stability. Various local stability criteria have been discussed in the literature (e.g., Bildsten 1998), in which one considers the properties of the gas at a single depth. FL87 proposed a global criterion involving an integral over the entire layer. While an improvement, this approach is still unsatisfactory since the authors assumed a constant temperature perturbation throughout the layer.

We have carried out a full linear stability analysis of the accretion layer. We start with the steady state solution and assume that it is slightly perturbed, $Q(\Sigma) \rightarrow Q(\Sigma)+$ $Q^{\prime}(\Sigma) \exp (\gamma t)$, where $Q$ corresponds to each of our five variables, and the perturbations $Q^{\prime}(\Sigma)$ are taken to be small. We linearize the five equations described earlier (three of which include time derivatives), apply the boundary conditions, and solve for the eigenvalue $\gamma$. We obtain a large number of solutions for $\gamma$ (technically, there is an infinite number since we are dealing with continuous functions), many of which have both a real and imaginary part. We consider the accretion layer to be unstable if any eigenvalue $\gamma$ has a real part (growth rate) greater than the characteristic accretion rate $\gamma_{a c c}=\dot{\Sigma} / \Sigma_{\max }$.

If the steady-state model is unstable according to the above criterion, it cannot accrete matter stably with the particular $\Sigma_{\max }$ and $\dot{\Sigma}$. Whether this instability manifests itself as a Type I burst depends on how the burning flame, once ignited, envelopes the surface of the star. This is as yet an unsolved problem, although Spitkovsky, Levin \& Ushomirsky (2002) present a possible solution. In the following we assume that the instability will grow rapidly to the nonlinear regime and that the system will indeed exhibit Type I bursts.

\section{Results}

Figure 1 shows results for solar composition material $\left(X_{0}=0.7, Y_{0}=0.27, Z_{0}=0.03\right)$ accreting on two kinds of compact stars. 

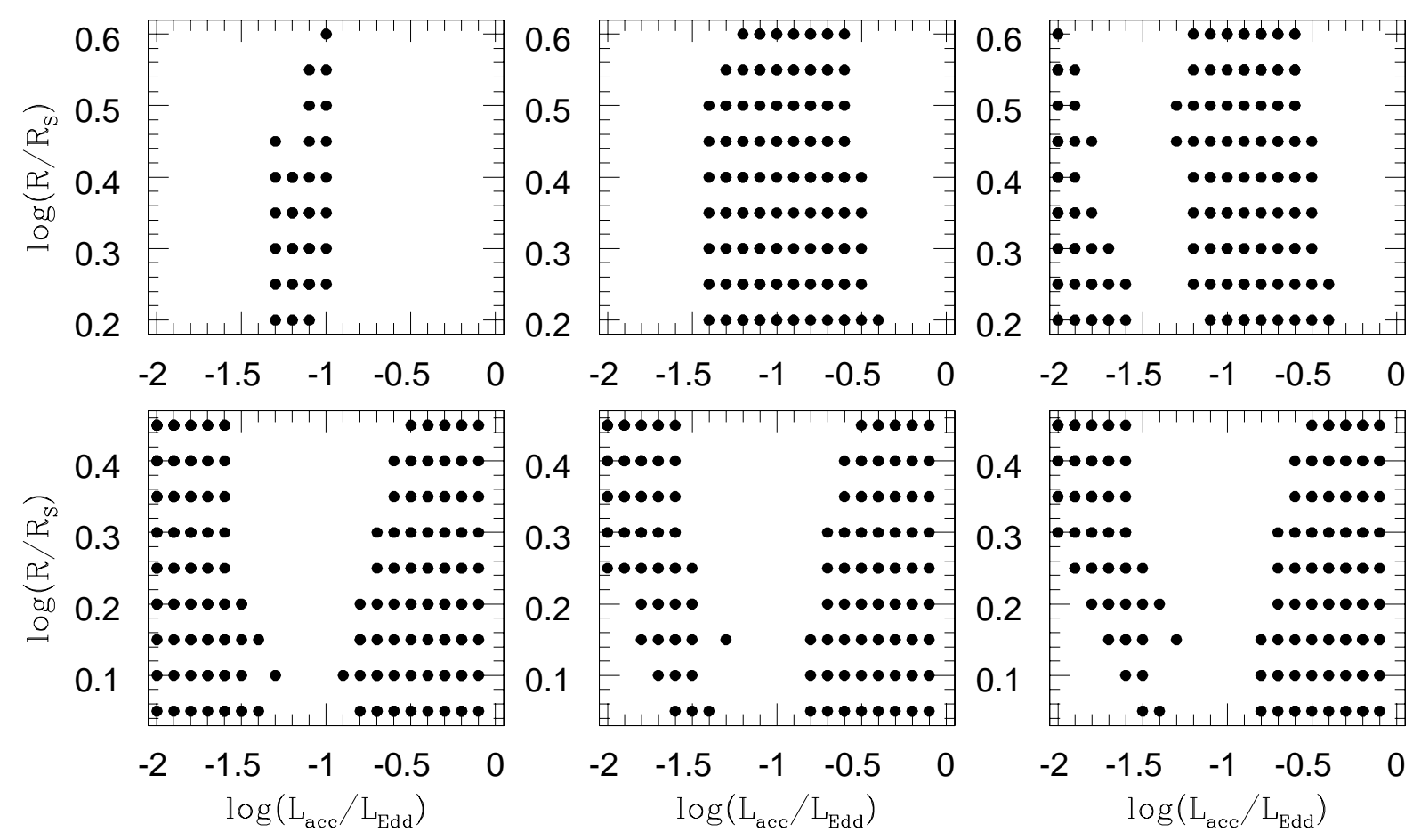

Fig. 1.- Regions of instability, shown by dots, as a function of accretion luminosity and stellar radius. Top left: $1.4 M_{\odot}$ NS with a base temperature $T_{i n}=10^{8.5} \mathrm{~K}$. Top center: $T_{\text {in }}=10^{8} \mathrm{~K}$. Top right: $T_{i n}=10^{7.5} \mathrm{~K}$. Bottom left: $10 M_{\odot} \mathrm{BH}$ candidate with a surface, and a base temperature $T_{i n}=10^{7.5} \mathrm{~K}$. Bottom center: $T_{i n}=10^{7} \mathrm{~K}$. Bottom right: $T_{i n}=10^{6.5} \mathrm{~K}$. 
The top three panels correspond to an accreting NS of mass $1.4 M_{\odot}$. We consider a range of accretion rates, parameterized by the ratio $L_{a c c} / L_{E d d}$, where we take the Eddington luminosity to be $L_{E d d}=4 \pi G M c / \kappa_{e s}$ with $\kappa_{e s}=0.4 \mathrm{~cm}^{2} \mathrm{~g}^{-1}$. We also consider a range of radii for the NS: $\log \left(R / R_{S}\right)=0.2-0.6(R=6.5-16 \mathrm{~km})$. For each choice of $L_{a c c} / L_{E d d}$ and $R / R_{S}$, we try three values of the surface mass density of the accreted layer: $\Sigma_{\max }=$ $10^{9}, 10^{10}, 10^{11} \mathrm{~g} \mathrm{~cm}^{-2}$. If any of the three cases is unstable, i.e., if it has any eigenvalue with $\operatorname{Re}(\gamma)>\gamma_{a c c}$, then we claim that the system will exhibit instabilities that may grow into Type I bursts.

The results presented correspond to three choices of the temperature at the base of the accreted layer: $T_{i n}=10^{8.5}, 10^{8}$ and $10^{7.5} \mathrm{~K}$ from left to right. In this Letter we are primarily interested in transient LMXBs. Since these sources have very low luminosities in quiescence $\left(L_{X}<10^{33} \mathrm{erg} \mathrm{s}^{-1}\right)$, the core temperatures of the NSs are likely to be $T_{\text {in }} \lesssim 10^{8} \mathrm{~K}$ (Possenti et al. 2001; Brown, Bildsten \& Chang 2002).

The calculations shown in Fig. 1 indicate that NSs are unstable to bursts for a wide range of $T_{i n}$, but that the width of the instability strip (as a function of $L_{a c c}$ ) is less for higher values of $T_{i n}$. The reason for this is clear from the analysis of P82 who showed that when the flux escaping from the stellar core into the accretion layer increases (which happens when $T_{i n}$ increases), bursting behavior is restricted to a smaller range of $\dot{\Sigma}$.

In the case of transient NS LMXBs, we expect $T_{\text {in }}$ to be $\lesssim 10^{8} \mathrm{~K}$. Fig. 1 indicates that these sources should be unstable to bursts for accretion luminosities up to $L_{a c c} \approx 0.3 L_{E d d}$. The predicted luminosity limit is generally consistent with observations; the only NSs that are known not to burst are bright $\mathrm{Z}$ sources with $L_{a c c} \rightarrow L_{E d d}$ (e.g., Matsuba et al. 1995), and X-ray pulsars. Although the latter are significantly less luminous than Eddington, they accrete effectively at close to the Eddington rate since the accreting matter is channeled onto a small area on the NS surface by strong magnetic fields (see Lamb 2000 for a detailed discussion of this argument).

Below $L_{a c c} \sim 0.3 L_{E d d}$, the instability is initially of a mixed form in which a He-burning instability triggers a burst in which $\mathrm{H}$ and He both burn explosively. At lower luminosities, nearly all the $\mathrm{H}$ is burned steadily and the instability corresponds to a pure He burst. These results are consistent with previous work (e.g., Bildsten 1998). The calculations indicate that bursting behavior cuts off below an accretion luminosity $L_{a c c} \sim 10^{-1.5} L_{E d d}$. The cutoff is the result of the restriction $\Sigma_{\max } \leq 10^{11} \mathrm{~g} \mathrm{~cm}^{-2}$ in our models. Systems with luminosities below our cutoff are still unstable to He bursts, but only at extremely high column density $\Sigma_{\text {max }}$. We have ignored such bursts since the recurrence time $t_{r e c}$ is too long to be of interest for observations of transient X-ray binaries. 
For $T_{i n}=10^{7.5} \mathrm{~K}$, there is a second instability strip at low luminosities $L_{\text {acc }} \sim 10^{-2} L_{E d d}$. This strip corresponds to pure $\mathrm{H}$ bursts; the possibility of such bursts was first noted by Fujimoto et al. (1987). An interesting difference between the two instability strips is that the strip on the right generally has complex eigenvalues $\gamma$ for the unstable modes while that on the left has real eigenvalues. As explained above, the gap between the two strips is real if we restrict ourselves to reasonable values of $\Sigma_{\max }$ and $t_{r e c}$. We are not aware of clear observational evidence for or against the gap, but such evidence could be searched for in future observations.

The bottom three panels in Fig. 1 show results for a $10 M_{\odot} \mathrm{BH}$ candidate with a surface. The three panels correspond to different choices of the base temperature: $T_{i n}=10^{7.5}, 10^{7}$ and $10^{6.5} \mathrm{~K}$ from left to right. The particular choices of $T_{i n}$ are motivated by the extraordinarily low quiescent luminosities of many transient BH LMXBs $\left(L_{X}<10^{31} \mathrm{erg} \mathrm{s}^{-1}\right.$, Narayan et al. 2001; a few BH LMXBs are brighter than this limit, but even these are not likely to have $\left.T_{\text {in }} \gtrsim 10^{7.5} \mathrm{~K}\right)$. We consider stellar radii in the range $\log \left(R / R_{S}\right)=0.05-0.45$, corresponding to $R$ between $(9 / 8) R_{S}$ and about $3 R_{S}$, a factor of nearly twenty in the surface gravity. The choice $(9 / 8) R_{S}$ corresponds to the smallest radius in general relativity for an object whose density either decreases or remains level with increasing radius (ST83).

The calculations indicate that BH candidates with surfaces are not very different from NSs in their bursting behavior. Except for a modest rightward shift of the positions of the instability zones, the results in the lower three panels are quite similar to those for a NS with a similarly low value of $T_{i n}$ (upper right panel). As in the case of NSs, bursts are expected for $\mathrm{BH}$ candidates even for larger values of $T_{i n}$ than we have considered, except that the instability strip becomes narrower when $T_{\text {in }}$ exceeds $10^{8} \mathrm{~K}$. We conclude that $\mathrm{BH}$ candidates are as prone to the instabilities that lead to Type I bursts as NSs are. The absence of bursts in BH LMXBs is thus highly significant and argues for the lack of surfaces in these systems.

Although our analysis is for a spherical, non-rotating $10 M_{\odot}$ star, the results are not expected to differ greatly for a rotating object. The effective surface gravity and accretion rate vary by only a factor of $2-3$ from pole to equator even for a maximally rotating oblate ellipsoid that is on the verge of the secular triaxial instability; furthermore, the dependence on compactness and equation of state is small (see Gondek-Rosinska \& Gourgoulhon 2002; ST83). In comparison, Fig. 1 shows that bursts are present for a wide range of surface gravity $g$ (factor of 20) and accretion rate $\dot{\Sigma}$ (factor of 100). The rather modest variation of $g$ and $\dot{\Sigma}$ with latitude in a rotating star is thus not likely to have an effect.

Because our model focuses only on the most important physical effects, and neglects many details, the exact positions of the instability strips in Fig. 1 may be uncertain at the level of say a factor of two in accretion luminosity. We believe, however, that the overall 
pattern of instability we have computed is fairly robust. Also, the calculations we present here do not directly predict what kind of bursts are produced. We leave this for a more detailed paper, but briefly, we find that for NSs with $L_{a c c} \sim 0.1 L_{E d d}$, bursts have durations of a few seconds and $t_{r e c} \sim 10$ hours. For lower $L_{a c c}$, as is well-understood, bursts have longer $t_{r e c}$ and larger fluences. These results agree qualitatively with observations.

\section{Discussion}

It is clear from the theory of bursts (Bildsten 1998, and references therein) that bursting behavior is largely determined by the surface gravity $g$, the mass accretion rate, and the composition of the accreting material. Since these parameters are similar in NS and $\mathrm{BH}$ systems, the bursting behavior of the two should be similar. We have quantified this argument with a global linear stability analysis which represents a technical advance over previous calculations.

The results presented in Fig. 1 show that if $\mathrm{BH}$ candidates had surfaces they ought to experience thermonuclear instabilities as commonly as NSs do; by inference, they should have frequent Type I bursts. However, no BH candidate has exhibited Type I bursts. The most obvious explanation is that NSs have surfaces and $\mathrm{BH}$ candidates have event horizons (Menou 2001). If there is no surface, material cannot accumulate, and therefore cannot become hot or dense enough to trigger a thermonuclear explosion.

Before we can claim that this "proves" the reality of the event horizon, more work is needed. First, we need to show that the model is able to reproduce the more detailed features of Type I bursts as observed in NS LMXBs. The statistics of burst durations and recurrence times (e.g., van Paradijs, Penninx \& Lewin 1988) and the occurrence of oscillatory behavior in some systems (Revnivtsev et al. 2000) ought to appear naturally in a realistic model. Also, any NS systems that burst when they should not by our calculations, or do not burst when they should according to the model, need to be explained. Second, the role of the inner boundary condition needs to be explored in detail. In Fig. 1, we see that different choices of the base temperature for a BH candidate give similar results. We have tried other boundary conditions, and also tried changing the composition of the accreting gas. In all cases we find that the accumulating layer is unstable to bursts for a wide range of luminosity, both in $1.4 M_{\odot} \mathrm{NSs}$ and $10 M_{\odot}$ compact stars with surfaces. Finally, the difficult issue of flame propagation over the surface of the star once the instability has been triggered needs to be addressed (e.g., Lamb 2000, Spitkovsky et al. 2002); the effect of rotation, for instance, is presently unclear. 
We should caution that the analysis presented here assumes that the accumulating gas on the surface of a BH candidate behaves like normal matter, with nucleons and electrons; this is the case for standard models of strange stars and Q-stars (Glendenning 1997). Obviously, the argument becomes invalid if the properties of the gas change drastically, e.g., if the nuclei disappeared and were replaced by quarks. Whether such extreme changes are plausible remains to be seen. The density and pressure at the base of the bursting layer do not go above few $\times 10^{8} \mathrm{~g} \mathrm{~cm}^{-3}$ and $10^{26} \mathrm{erg} \mathrm{cm}^{-3}$ even in the most extreme cases we have considered. It is hard to imagine exotic physics being important under these conditions (Glendenning 1997).

On the observational front, we should check whether some NSs that lie within the unstable regions of Fig. 1 are stable to bursts. Any obvious large-scale disagreement between the observed burst behavior of NS LMXBs and the results presented here would indicate that the model is missing important physics. In the case of $\mathrm{BH}$ binaries, we should use observational data to derive quantitative limits on bursting activity. The transient $\mathrm{BH}$ LMXBs are particularly important for such work since they vary over a wide range of $L_{a c c}$ during their accretion outbursts (Tanaka \& Shibazaki 1996). If these sources have surfaces it is virtually impossible to arrange matters such that the objects have no bursts at all over the entire range of $L_{a c c}$. A firm demonstration that BH transients do not have Type I bursts would thus be a strong argument for the presence of event horizons in these systems.

We thank Andrew Cumming, Alex Ene, Kristen Menou, Bohdan Paczyński and Greg Ushomirsky for useful discussions, and two referees for useful comments. RN was supported in part by the W. M. Keck Foundation as a Keck Visiting Professor. RN's research was supported by NSF grant AST-9820686 and NASA grant NAG5-10780. JSH was supported by the Chandra Postdoctoral Fellowship Award \# PF0-10015 issued by the Chandra X-ray Observatory Center, which is operated by the Smithsonian Astrophysical Observatory for and on behalf of NASA under contract NAS8-39073. 


\section{REFERENCES}

Bildsten, L. 1998, in The Many Faces of Neutron Stars, eds R. Buccheri, J. van Paradijs, \& M. A. Alpar (Dordrecht: Kluwer), 419

Bildsten, L. \& Cumming, A. 1998, ApJ, 506, 842

Brown, E. F., Bildsten, L., \& Chang, P. 2002, ApJ, in press

Clayton, D. D. 1968, Principles of Stellar Evolution and Nucleosynthesis (New York: McGraw-Hill)

Fujimoto, M. Y., Hanawa, T., Iben, I., \& Richardson, M. B. 1984, ApJ, 278, 813

Fujimoto, M. Y., Hanawa, T., Iben, I., \& Richardson, M. B. 1987, ApJ, 315, 198

Fushiki, I., \& Lamb, D. Q. 1987, ApJ, 323, L55 (FL87)

Glendenning, N. K. 1997, Compact Stars, Nuclear Physics, Particle Physics, and General Relativity (Berlin: Springer)

Gondek-Rosinska, D. \& Gourgoulhon E. 2002, Phys. Rev. D, submitted (gr-qc/0205102)

Grindlay, J., et al. 1976, ApJ, 205, L127

Hansen, C. J., \& van Horn, H. M. 1975, ApJ, 195, 735

Heyl, J. S., \& Hernquist, L. 2001, MNRAS, 324, 292

Iben, I. 1975, ApJ, 196, 525

Joss, P. C. 1977, Nature, 270, 310

Lamb, D. Q. 2000, ApJS, 127, 395.

Lewin, W. H. G., van Paradijs, J., \& Taam, R.E. 1993, Sp. Sci. Rev., 62, 223

Mathews, G. J. \& Dietrich, F. S. 1984, ApJ, 287, 969

Matsuba, E., et al. 1995, PASJ, 47, 575

Menou, K. 2001, in Proc. 2nd KIA Astrophysics Workshop (astro-ph/0111469)

Narayan, R., Garcia, M. R., \& McClintock, J. E. 2001, in Proc. IX Marcel Grossmann Meeting, eds V. Gurzadyan, R. Jantzen, \& Ruffini (Singapore: World Scientific) (astro-ph/0107387) 
Paczyński, B. 1982, ApJ, 264, 282 (P82)

Possenti, A., Colpi, M., Page, D., \& Geppert, U. 2001, ASP Conf. Ser. 229: Evolution of Binary and Multiple Star Systems, 483

Revnivtsev, M., Churazov, E., Gilfanov, M., \& Sunyaev, R. 2001, A\&A, 372, 138

Schatz, H., Bildsten, L., Cumming, A., \& Wiescher, M. 1999, ApJ, 524, 1014

Shapiro, S. L. \& Teukolsky, S. A. 1983, Black Holes, White Dwarfs, and Neutron Stars (New York: Wiley-Interscience) (ST83)

Spitkovsky, A., Levin, Y., \& Ushomirsky, G. 2002, ApJ, 566, 1018

Strohmayer, T. E., Swank, J. H. \& Zhang, W. 1998, Nucl. Phys. B (Proc. Suppl.), 69, 129.

Taam, R. E., \& Picklum, R. E. 1978, ApJ, 224, 210

Taam, R. E., Woosley, S. E., \& Lamb, D. Q. 1996, ApJ, 459, 271

Tanaka, Y., \& Shibazaki, N. 1996, ARA\&A, 34, 607

van Paradijs, J., Penninx, W., \& Lewin, W. H. G. 1988, MNRAS, 233, 437

Woosley, S. E., \& Taam, R. E. 1976, Nature, 263, 101 